Research Article

\title{
The Adjuvant Activity of Epimedium Polysaccharide-Propolis Flavone Liposome on Enhancing Immune Responses to Inactivated Porcine Circovirus Vaccine in Mice
}

\author{
Yunpeng Fan, ${ }^{1}$ Liwei Guo, ${ }^{2}$ Weifeng Hou, ${ }^{1}$ Chao Guo, ${ }^{1}$ Weimin Zhang, \\ $\mathrm{Xia} \mathrm{Ma},{ }^{3}$ Lin $\mathrm{Ma}^{1}{ }^{1}$ and Xiaoping Song ${ }^{1}$ \\ ${ }^{1}$ College of Veterinary Medicine, Northwest A\&F University, Yangling, Shaanxi 712100, China \\ ${ }^{2}$ College of Animal Science, Yangtze University, Jingzhou 434023, China \\ ${ }^{3}$ Medicinal Engineering Department of Henan University of Animal Husbandry and Economy, Zhengzhou, Henan 450011, China
}

Correspondence should be addressed to Xiaoping Song; fanyunpeng83@163.com

Received 4 August 2015; Accepted 1 October 2015

Academic Editor: Kuttulebbai N. S. Sirajudeen

Copyright (C) 2015 Yunpeng Fan et al. This is an open access article distributed under the Creative Commons Attribution License, which permits unrestricted use, distribution, and reproduction in any medium, provided the original work is properly cited.

\begin{abstract}
Objectives. The adjuvant activity of Epimedium polysaccharide-propolis flavone liposome (EPL) was investigated in vitro and in vivo. Methods. In vitro, the effects of EPL at different concentrations on splenic lymphocytes proliferation and mRNA expression of IFN- $\gamma$ and IL- 6 were determined. In vivo, the adjuvant activities of EPL, EP, and mineral oil were compared in BALB/c mice through vaccination with inactivated porcine circovirus type 2 (PCV2) vaccine. Results. In vitro, EPL promoted lymphocytes proliferation and increased the mRNA expression of IFN- $\gamma$ and IL-6, and the effect was significantly better than EP at all concentrations. In vivo, EPL significantly promoted the lymphocytes proliferation and the secretion of cytokines and improved the killing activity of NK cells, PCV2-specific antibody titers, and the proportion of T-cell subgroups. The effects of EPL were significantly better than EP and oil adjuvant at most time points. Conclusion. EPL could significantly improve both PCV2-specific cellular and humoral immune responses, and its medium dose had the best efficacy. Therefore, EPL would be exploited in an effective immune adjuvant for inactivated PCV2 vaccine.
\end{abstract}

\section{Introduction}

Porcine circovirus (PCV) is a single-stranded DNA virus, which is one of the smallest animal viruses known to man [1]. There are two genotypes: PCV1 and PCV2. PCV2 possesses pathogenicity, which is the major pathogen for causing postweaning multisystemic wasting syndrome (PMWS) in piglets [2]. Studies show that PCV2 can lead to concurrent infection combined with other viruses such as porcine reproductive and respiratory syndrome virus and porcine parvovirus $[3,4]$. Moreover, PCV2 can infringe the lymphatic system of pig and thus induce the deficiency of lymphocytes and antigen presenting cells which result in the immunosuppression of body and easily cause secondary infection [5]. Therefore, how to effectively prevent and control PCV2 infection is the issue to be solved urgently in pig industry. PCV2 has become one of the research hotspots in the field of veterinary medicine at home and abroad.

For PCV2 disease, there is no effective medicine for its treatment in clinic. Vaccination is an effective way for preventing this disease. But the immune response induced by most vaccines is suboptimal without the help of adjuvants. Immune adjuvant plays an essential role in vaccine [6]. However, there are some deficiencies for commonly used adjuvant in clinic $[7,8]$. Therefore, it is particularly important to develop new types of immune adjuvants. Nowadays, the focus of drug research has shifted from synthetic drug to natural medicine, and the study of natural products has become a hotspot $[9,10]$.

Epimedium, a famous traditional Chinese herbal medicine, could significantly enhance the immune function of body. The polysaccharide from Epimedium has been 
proved to be the primary active compound responsible for the immunomodulatory effect of this drug [11]. Propolis has been widely used as the adjuvant of vaccines, because it could induce earlier immune response and longer protection period [12]. Its main compound is flavone. Our previous studies proved that the prescription of Epimedium polysaccharide and propolis flavone (EP) could synergistically enhance humoral immunity and cellular immunity of organism and improve the immune effect of inactivated avian influenza and Newcastle disease vaccine $[13,14]$.

Studies have confirmed that liposome could improve the bioavailability of drugs [15]. Our previous studies have proved that the immunoenhancing activity of the effective constituents from plants was significantly improved after being made into liposome $[16,17]$. Recently, we have found that liposome also could further improve the immune enhancement activity of EP and improve the immune response of Newcastle disease vaccine $[18,19]$. Therefore, we speculate EP liposome (EPL) could also enhance the immune effect of PCV2 vaccine. In the present study, the adjuvant activity of EPL against inactivated PCV2 vaccine was investigated. The purpose of this study was to investigate whether EPL could enhance the immune response of PCV2 vaccine and select the best dosage for clinical use. This study will provide theoretical support for developing a new type of adjuvant for inactivated PCV2 vaccine.

\section{Materials and Methods}

2.1. Reagents. Lymphocyte separation medium was obtained from Tianjin Hanyang Biologicals Technology Co. Ltd. RPMI-1640 and fetal bovine serum (FBS) were obtained from Hyclone (USA). Trypsin (Amresco) was dissolved in $0.25 \%$ with calcium and magnesium-free phosphate-buffered saline (CMF-PBS, pH 7.2). Goat anti-mouse monoclonal antiCD4-FITC and anti-CD8-PE antibodies were supplied by BioLegend Inc. (USA). The 3-(4,5-dimethylthiazol-2-yl)-2,5diphenyltetrazolium bromide (MTT) and dimethyl sulfoxide (DSMO) were purchased from Sigma-Aldrich Co. LLC. All the other chemicals used were of analytical grade.

2.2. Preparation of EPL. Epimedium polysaccharide-propolis flavone liposome (EPL) was prepared in our laboratory according to previous method [19]. EPL solution with a concentration of $2.0 \mathrm{mg} \cdot \mathrm{mL}^{-1}$ was prepared by dissolving in PBS. The solution was sterilized by a $0.22 \mu \mathrm{m}$ Millipore filter. The endotoxin amount was up to the standard of the Chinese Veterinary Pharmacopoeia (less than $0.5 \mathrm{EU} / \mathrm{mL}$ ). Then, it is stored at $4^{\circ} \mathrm{C}$ for the test.

2.3. Vaccine and Virus. Inactivated PCV2 (SH strain) was offered by China Institute of Veterinary Drug Control. The inactivated virus was used as vaccine antigen. EPL adjuvant vaccine at three doses contained $2.0,1.5$, and $1.0 \mathrm{mg}$ of EPL, respectively, in $1 \mathrm{~mL}$ of vaccine. EP adjuvant vaccine contained $2.0 \mathrm{mg}$ of $\mathrm{EP}$ in $1 \mathrm{~mL}$ of vaccine. Oil adjuvant (OA) vaccine was prepared by emulsification with mineral oil according to the literature [20]. All the vaccines contained the same amount of PCV2.

2.4. Measurement of Splenic Lymphocytes Proliferation In Vitro. The splenic lymphocytes were prepared according to previous report [21]. $100 \mu \mathrm{L} /$ well of splenic lymphocytes $\left(5.0 \times 10^{6}\right.$ cells $\left./ \mathrm{mL}\right)$ was plated on a 96 -well culture plate. Then, $80 \mu \mathrm{L}$ of EPL, EP, and blank liposome (BL) in different concentrations was added into each well, respectively. Finally, PHA, LPS, or medium was added for giving a final volume of $200 \mu \mathrm{L}$. The final concentration of PHA reached $20 \mu \mathrm{g} \cdot \mathrm{mL}^{-1}$, and LPS reached $10 \mu \mathrm{g} \cdot \mathrm{mL}^{-1}$. Each concentration of sample was repeated four times. The plates were cultured at $37^{\circ} \mathrm{C}$ in a $5 \% \mathrm{CO}_{2}$ humidified incubator for $48 \mathrm{~h} .20 \mu \mathrm{L}$ of MTT solution $\left(5 \mu \mathrm{g} \cdot \mathrm{mL}^{-1}\right)$ was added to each well and incubated for an additional $4 \mathrm{~h}$. The supernatant was removed and $100 \mu \mathrm{L}$ of DMSO was added. The absorbance of cells was evaluated by using microplate reader (Bio-Rad, USA) at a wavelength of $570 \mathrm{~nm}$ ( $A_{570}$ value). The stimulation index (SI) was calculated as the index of splenic lymphocytes proliferation based on the following formula: SI = the absorbance value of drug group/the absorbance value of cell control group [22].

2.5. Measurement of IFN- $\gamma$ and IL-6 mRNA Expression In Vitro. The method for preparing splenic lymphocytes was performed according to previous report [21]. After being treated with EPL for $48 \mathrm{~h}$, the RNA of lymphocytes was extracted. The reaction condition was carried out according to the manufacturer's instruction. Real-time quantitative PCR was utilized on the ABI 7300 (PE Applied Biosystems, USA) using SYBR premix Taq Kit (Takara, China). The primers for target gene and GAPDH were listed as follows: IFN- $\gamma$ (183 bp), forward: $5^{\prime}$-CGGCACAGTCATTGAAAGCCTA-3', reverse: $5^{\prime}$-GTTGCTGATGGCCTGATTGTC3'; IL-6 (141 bp), forward: 5'-GAGGATACCACTCCCAACAGACC-3', reverse: $5^{\prime}$-AAGTGCATCATCGTTGTTCATACA-3'; GAPDH (108 bp), forward: 5' -AAATGGTGAAGGTCGGTGTG-3', reverse: $5^{\prime}$-TGAAGGGGTCGTTGATGG$3^{\prime}$. Amplification was carried out in a final volume of $25 \mu \mathrm{L}$. The following experimental run protocol was used: denaturation $\left(95^{\circ} \mathrm{C}\right.$ for $\left.30 \mathrm{~s}\right)$, annealing $\left(60^{\circ} \mathrm{C}\right.$ for $\left.30 \mathrm{~s}\right)$, and elongation $\left(72^{\circ} \mathrm{C}\right.$ for $\left.30 \mathrm{~s}\right)$. The program was performed with 40 PCR cycles. The relative quantification among specimens was calculated according to $2^{-\Delta \Delta \mathrm{Ct}}$ method [23].

\subsection{The Design of Experiment In Vivo}

2.6.1. Animals. Six-week-old BALB/c mice weighing 23-25 g were purchased from Laboratory Animal Center of Fourth Military Medical University (Shaanxi, China). They were fed in an environmentally controlled animal facility maintained under controlled conditions with humidity of $50 \pm 10 \%$, temperature of $24 \pm 1^{\circ} \mathrm{C}$, and a 12/12 h light/dark cycle. All the procedures were in strict accordance with the National Institutes of Health Guide for the Care and Use of Experimental Animals approved by the Animal Ethics Committee of 
Northwest A\&F University. All efforts were made to minimize the number of animals used and their suffering.

2.6.2. Adjuvant Activity. Three hundred and sixty BALB/c mice were randomly divided into six groups with 60 mice in each. The mice in three dosage groups (2.0, 1.5, and $1.0 \mathrm{mg} \cdot \mathrm{mL}^{-1}$ ) of EPL were subcutaneously injected with $0.3 \mathrm{~mL}$ of EPL vaccine, respectively. In EP and OA groups, the mice were injected with $0.3 \mathrm{~mL}$ of $\mathrm{EP}$ vaccine and $\mathrm{OA}$ vaccine, respectively. In blank control (BC) group, the mice were injected with $0.3 \mathrm{~mL}$ of physiological saline. The mice were repeatedly vaccinated after two weeks. On days 7, 14, 21,28 , and 35 after the first vaccination, the blood samples of six mice were collected randomly from each group for measuring peripheral lymphocytes proliferation by MTT method, specific PCV2 antibody titers, and the contents of cytokines (IFN- $\gamma$ and IL-6) in serum by ELISA. At the same time, splenocytes were harvested for determining the killing activity of NK cells. In addition, the blood samples of four mice were collected randomly from each group for assaying $\mathrm{CD}^{+}$and $\mathrm{CD}^{+}$T-lymphocytes subpopulations by flow cytometry on days 21, 28, and 35 .

2.6.3. Measurement of Cytokines Levels. The contents of IFN- $\gamma$ and IL- 6 in serum were assayed by indirect ELISA according to the manufacturer's instructions (Biosamite Biotechnology Co. Ltd., Shanghai, China). The absorbance was measured by using a microplate reader at $450 \mathrm{~nm}$. The contents of IFN- $\gamma$ and IL- 6 were calculated according to the standard curve.

2.6.4. Measurement of NK Cell Activity. The killing activity of NK cell in splenocytes was determined by MTT method [24]. Briefly, SP2/0 cells were used as target cells, which were adjusted to $2.0 \times 10^{4}$ cells $/ \mathrm{mL}$, and seeded in 96-well culture plates with $100 \mu \mathrm{L} /$ well. Then, splenocytes were prepared and adjusted to $1.0 \times 10^{6}$ cells $/ \mathrm{mL}$ and added to a final volume of $200 \mu \mathrm{L}$. The plates were incubated for $20 \mathrm{~h}$ at $37^{\circ} \mathrm{C}$ in $5 \% \mathrm{CO}_{2}$ humidified incubator. $50 \mu \mathrm{L}$ of MTT solution $\left(5 \mathrm{mg} \cdot \mathrm{mL}^{-1}\right)$ was added to each well and the plate was incubated for another $4 \mathrm{~h}$. The absorbance of cells was evaluated at a wavelength of $630 \mathrm{~nm}$ ( $A_{630}$ value). NK cell activity was calculated as the following equation: NK cell activity $(\%)=\left(t A_{630}-\right.$ $\left.\left(s A_{630}-b A_{630}\right)\right) / t A_{630} \times 100 \%$, where $t A_{630}$ is the absorbance of the target cells control, $s A_{630}$ is the absorbance of test samples, and $b A_{630}$ is the absorbance of blank cells control [24].

2.6.5. Measurement of PCV2-Specific Antibody Titers. PCV2specific IgG, IgG1, and IgG2a antibodies titers were measured by indirect ELISA according to the manufacturer's instructions. The absorbance was measured in a microplate reader at $630 \mathrm{~nm}$ [25]. ELISA assays were performed on the same day for all of the samples.

2.6.6. Measurement of $\mathrm{CD}^{+}$and $C D 8^{+}$T-Lymphocytes Percentages. Peripheral blood lymphocytes were isolated according to the previous method [26]. The cells were adjusted to $5.0 \times 10^{6} \cdot \mathrm{mL}^{-1}$. Then, lymphocytes were incubated at $4^{\circ} \mathrm{C}$ for $30 \mathrm{~min}$ in the dark with FITC-conjugated anti-mouse CD4 and PE-conjugated anti-mouse CD8 monoclonal antibodies. After being centrifuged at $3000 \times \mathrm{g}$ for 5 min at $4^{\circ} \mathrm{C}$, the cells were washed twice with ice-cold PBS and resuspended in $0.5 \mathrm{~mL}$ of PBS. The proportions of $\mathrm{CD}^{+}$ and $\mathrm{CD} 8^{+}$T-lymphocytes were assayed by flow cytometry.

2.7. Statistical Analysis. The data were expressed as mean \pm standard deviation (SD). Duncan's multiple range test was used to determine the differences among groups with the software SPSS 19.0. $P$ values of less than 0.05 were considered to be statistically significant.

\section{Result}

3.1. Effect of EPL on Splenic Lymphocyte Proliferation In Vitro. The stimulation indexes (SI) in each group are illustrated in Figure 1. In single stimulation of EPL and EP on splenic lymphocyte, the SI in EPL group was higher than those in $\mathrm{BL}$ and cell control (CC) groups under all concentrations $(P<0.05)$. In addition, the SI in EPL group was significantly higher than those in EP group at all concentrations $(P<$ 0.05) (Figure 1(a)). After synergistic stimulation with PHA, the SI in EPL group was the highest and was significantly higher than those in EP group at $8-1 \mu \mathrm{g} \cdot \mathrm{mL}^{-1}(P<0.05)$ (Figure 1(b)). After synergistic stimulation with LPS, the SI in EPL group was the highest and was significantly higher than those in EP group at all concentrations $(P<0.05)$ (Figure 1(c)).

3.2. Effect of EPL on the mRNA Expression of IFN- $\gamma$ and IL-6 In Vitro. The effects of EPL on the mRNA expression of IFN$\gamma$ and IL-6 in splenocytes are illustrated in Figure 2. At 16$1 \mu \mathrm{g} \cdot \mathrm{mL}^{-1}$, the expression levels of IFN- $\gamma$ and IL- 6 in EPL and EP groups were significantly higher than those in BL and CC groups $(P<0.05)$. In addition, the expression levels of IFN- $\gamma$ and IL-6 in EPL group were significantly higher than that in EP group at all concentrations $(P<0.05)$.

\subsection{The Dynamic Changes of T-Lymphocytes Proliferation In} Vivo. The SI in each group is illustrated in Figure 3. At all time points after the first vaccination, the SI in EPL at three doses and EP groups were significantly higher than those in BC group $(P<0.05)$. On days $14-35$, the $\mathrm{SI}$ in $\mathrm{EPL}_{\mathrm{H}}$ and $\mathrm{EPL}_{\mathrm{M}}$ groups were significantly higher than those in $\mathrm{EPL}_{\mathrm{L}}$ and EP groups $(P<0.05)$. At all time points, the SI in $\mathrm{EPL}_{\mathrm{H}}$ and $\mathrm{EPL}_{\mathrm{M}}$ groups were significantly higher than those in $\mathrm{OA}$ group $(P<0.05)$.

3.4. Effect of EPL on the Secretion of IFN- $\gamma$ and IL-6 in Serum. Effects of EPL on the secretion of IFN- $\gamma$ and IL- 6 are shown in Figure 4. On days 14-35, the IFN- $\gamma$ contents in $\mathrm{EPL}_{\mathrm{H}}$ and $\mathrm{EPL}_{\mathrm{M}}$ groups were significantly higher than those in $\mathrm{EPL}_{\mathrm{L}}$ and EP groups $(P<0.05)$. At all time points, the IFN- $\gamma$ 


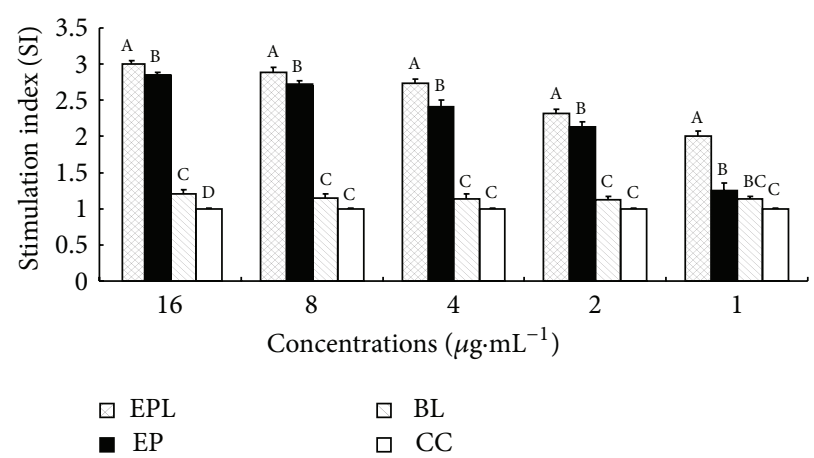

(a)

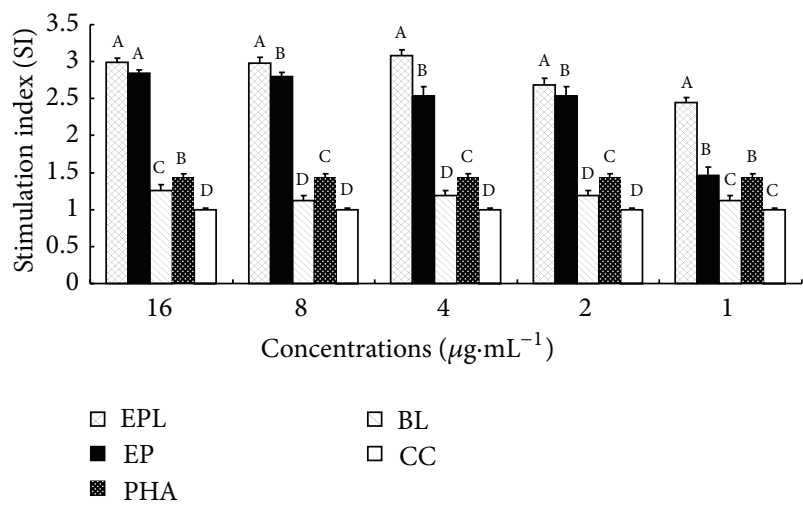

(b)

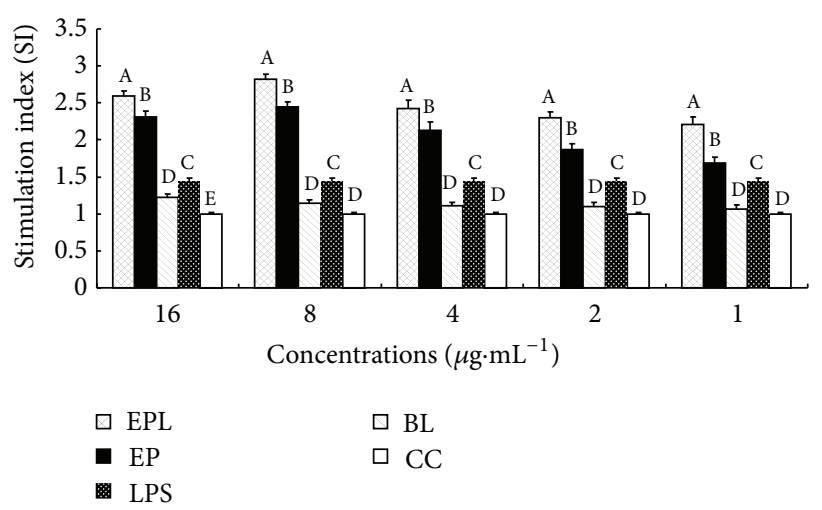

(c)

FIGURE 1: Effect of EPL on splenic lymphocyte proliferation in vitro (SI). (a) The single stimulation of drugs. (b) The synergistic stimulation of drugs with PHA. (c) The synergistic stimulation of drugs with LPS. ${ }^{\mathrm{A}-\mathrm{D}}$ Bars in the figure without the same superscripts differ significantly $(P<0.05)$

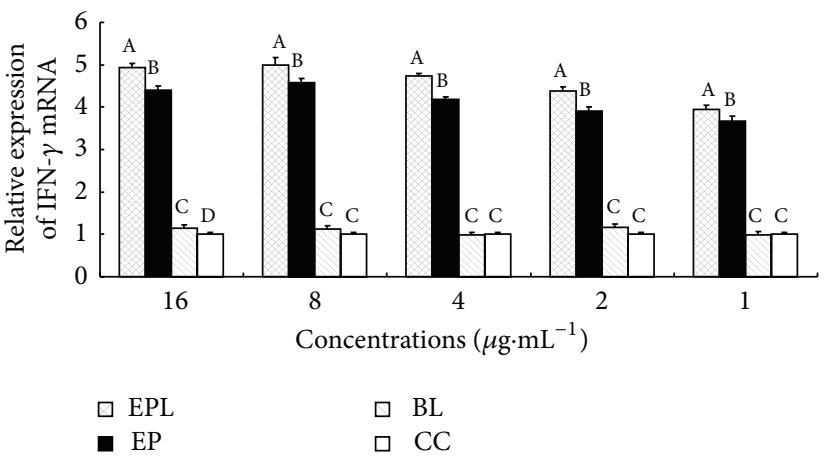

(a)

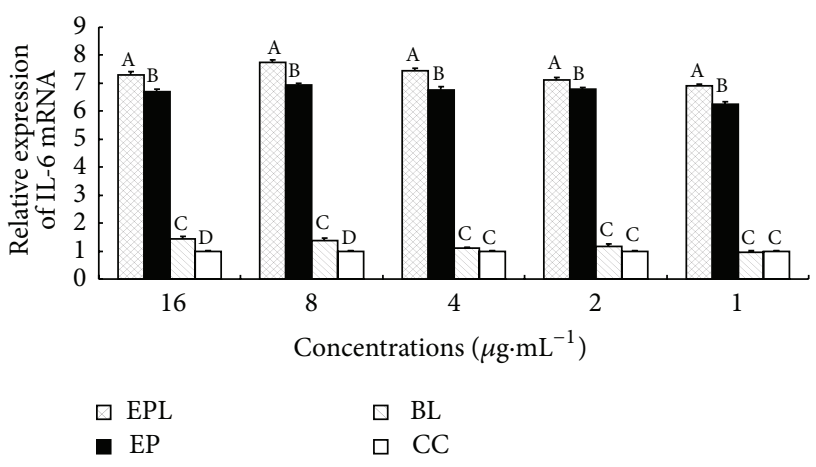

(b)

FiguRE 2: Effect of EPL on the mRNA expression of IFN- $\gamma$ and IL-6. (a) The mRNA expression of IFN- $\gamma$. (b) The mRNA expression of IL-6. Bars in the figure without the same superscripts differ significantly $(P<0.05)$.

contents in $\mathrm{EPL}_{\mathrm{M}}$ group were significantly higher than those in OA group $(P<0.05)$. The IFN- $\gamma$ contents in the $\mathrm{EPL}_{\mathrm{H}}$ group were significantly higher than those in OA group on days $7,14,21$, and $35(P<0.05)$ (Figure $4(a))$. On days 14-35, the IL-6 contents in $\mathrm{EPL}_{\mathrm{H}}$ and $\mathrm{EPL}_{\mathrm{M}}$ group were significantly higher than those in $\mathrm{EPL}_{\mathrm{L}}, \mathrm{EP}$, and $\mathrm{BC}$ groups $(P<0.05)$. In addition, at all time points, the IL- 6 contents in $\mathrm{EPL}_{\mathrm{H}}$ and
$\mathrm{EPL}_{\mathrm{M}}$ groups were also significantly higher than those in $\mathrm{OA}$ group $(P<0.05)$ (Figure 4(b)).

3.5. Effect of EPL on the Activity of NK Cells In Vivo. The effects of EPL on NK cell activity were shown in Figure 5. On days 14-35, the activities of NK cells in $\mathrm{EPL}_{\mathrm{H}}, \mathrm{EPL}_{\mathrm{M}}, \mathrm{EPL}_{\mathrm{L}}$, $\mathrm{EP}$, and $\mathrm{OA}$ groups were significantly higher than those in $\mathrm{BC}$ 


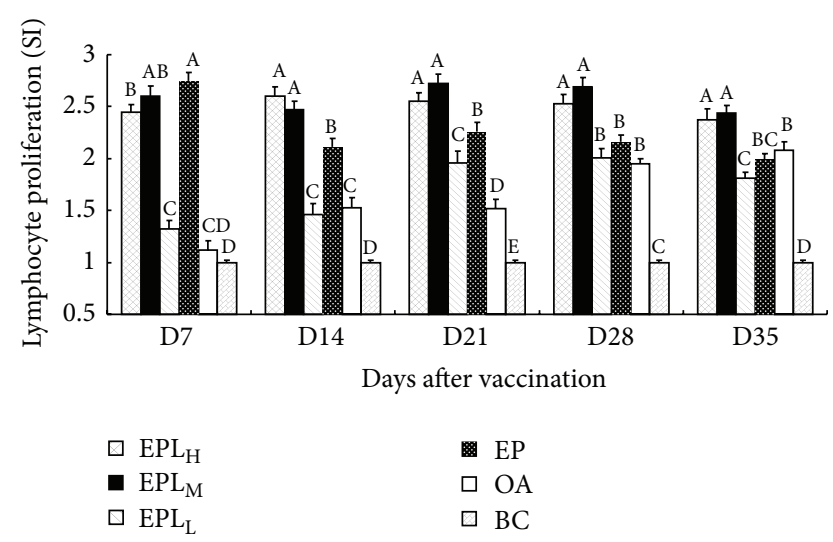

FIgURE 3: The effects of EPI on T-lymphocytes proliferation in the immunized mice (SI). Bars in the same day without the same superscripts differ significantly $(P<0.05)$. H: high dose; M: medium dose; L: low dose.

group ( $P<0.05)$. On days 14-35, the activities of NK cells in $\mathrm{EPL}_{\mathrm{H}}$ and $\mathrm{EPL}_{\mathrm{M}}$ groups were significantly higher than those in $\mathrm{EPL}_{\mathrm{L}}, \mathrm{EP}$, and OA groups $(P<0.05)$.

3.6. Effect of EPL on the PCV2-Specific Titers. The effect of EPL on the PCV2-specific IgG, IgG1, and IgG2a titers was shown in Figure 6. On days 14-35, the IgG titers in $\mathrm{EPL}_{\mathrm{H}}$ and $\mathrm{EPL}_{\mathrm{M}}$ groups were significantly higher than those in other groups $(P<0.05)$ (Figure 6(a)). On days 14-35, the IgG1 titers in $\mathrm{EPL}_{\mathrm{M}}$ group were significantly higher than those in $\mathrm{EPL}_{\mathrm{L}}$ and $\mathrm{EP}$ groups; $\mathrm{EPL}_{\mathrm{H}}$ group was higher than EP group, and the differences were significant on days 21, 28, and $35(P<0.05)$. In addition, at all time points, the IgG1 titers in $\mathrm{EPL}_{\mathrm{M}}$ group were significantly higher than those in OA group $(P<0.05)$. $\mathrm{EPL}_{\mathrm{H}}$ was higher than $\mathrm{OA}$, and the differences were significant on days 14,28 , and $35(P<0.05)$ (Figure 6(b)). On days 14-35, the IgG2a titers in $\mathrm{EPL}_{\mathrm{H}}$ and $\mathrm{EPL}_{\mathrm{M}}$ groups were significantly higher than those in $\mathrm{EPL}_{\mathrm{L}}$, EP, and OA groups $(P<0.05)$ (Figure $6(\mathrm{c}))$.

\subsection{The Effects of EPI on the Percentages of $C D 4^{+}$and $C D 8^{+} T$ -} Cells. The changes of $\mathrm{CD} 4^{+}$and $\mathrm{CD}^{+} \mathrm{T}$-cells proportions are shown in Figure 7. On days 21-35, the proportions of $\mathrm{CD}^{+}$ T-cells in $\mathrm{EPL}_{\mathrm{H}}$ group were $39.45 \%, 40.60 \%$, and $37.53 \%$, in $\mathrm{EPL}_{\mathrm{M}}$ group were $39.00 \%, 43.46 \%$, and $39.37 \%$, in EP group were $30.14 \%, 35.27 \%$, and $26.14 \%$, and in OA group were $26.55 \%, 30.62 \%$, and $25.88 \%$, respectively. On days $21-35$, the proportions of $\mathrm{CD}^{+}{ }^{+} \mathrm{T}$-cells in $\mathrm{EPL}_{\mathrm{H}}$ and $\mathrm{EPL}_{\mathrm{M}}$ groups were significantly higher than those in EP group $(P<0.05)$. In addition, at all time points, the proportions of $\mathrm{CD} 4^{+} \mathrm{T}$-cells in $\mathrm{EPL}_{\mathrm{H}}$ and $\mathrm{EPL}_{\mathrm{M}}$ groups were significantly higher than those in OA group $(P<0.05)$ (Figure $7(\mathrm{a}))$. On days $21-35$, the proportions of $\mathrm{CD}^{+} \mathrm{T}$-cells in $\mathrm{EPL}_{\mathrm{H}}$ group were $30.04 \%$, $33.17 \%$, and $31.39 \%$, in $\mathrm{EPL}_{\mathrm{M}}$ group were $29.31 \%, 34.68 \%$, and $33.90 \%$, in EP group were $26.51 \%, 30.26 \%$, and $23.50 \%$, and in OA group were $23.15 \%, 27.26 \%$, and $28.22 \%$, respectively. On days 21-35, the proportion of $\mathrm{CD}^{+}{ }^{+} \mathrm{T}$-cells in $\mathrm{EPL}_{\mathrm{H}}$ group was significantly higher than those in $\mathrm{EP}, \mathrm{EPL}_{\mathrm{L}}$, and $\mathrm{BC}$ groups $(P<0.05)$. At three time points, the proportion of $\mathrm{CD}^{+}$ T-cells in $\mathrm{EPL}_{\mathrm{M}}$ group was higher than those in EP group, and the differences were significant on days 28 and $35(P<$ 0.05). In addition, the proportions of $\mathrm{CD}^{+}{ }^{+}$-cells in $\mathrm{EPL}_{\mathrm{H}}$ and $\mathrm{EPL}_{\mathrm{M}}$ groups were significantly higher than those in $\mathrm{OA}$ group at all time points $(P<0.05)$ (Figure $7(b))$.

\section{Discussion}

The immune system of body mainly consists of cell immunity and humoral immunity [27]. The capacity to induce effective cellular immunity could be determined by $\mathrm{T}$ - and $\mathrm{B}-$ lymphocytes proliferation $[28,29]$. In order to investigate the effect of EPL on enhancing cellular immunity, the splenic lymphocytes proliferation was firstly measured in vitro. The results showed that EPL could significantly promote the splenic lymphocytes proliferation when stimulated with PHA/LPS or single stimulation at $16-1 \mu \mathrm{g} \cdot \mathrm{mL}^{-1}$ compared with EP. In addition, the experimental results in vivo also showed that EPL at high and medium doses could significantly promote peripheral lymphocytes proliferation at most time points. It is suggested that EPL could significantly enhance the activation of T- and B-cells in immunized mice. T-lymphocyte can be divided into $\mathrm{CD} 4^{+}$and $\mathrm{CD} 8^{+}$subsets [30]. The number of $\mathrm{CD}^{+}{ }^{+} \mathrm{T}$-cells and $\mathrm{CD} 8^{+} \mathrm{T}$-cells is one of the bases in assessing the immune status of organism [31]. The function of cellular immunity and humoral immunity will be improved along with the increasing counts of $\mathrm{CD}^{+}$and $\mathrm{CD}^{+}{ }^{+} \mathrm{T}$-cells [32]. In order to further investigate the effect of EPL on the cellular immunity, the expressions of $\mathrm{CD}^{+}$ and $\mathrm{CD}^{+}$T-lymphocytes subpopulations were determined. The results showed that the proportions of $\mathrm{CD} 4^{+}$and $\mathrm{CD} 8^{+}$ T-cells in EPL at medium dose group were significantly higher than those in EP group at all time points, which suggested that EPL could promote lymphocytes proliferation and increase the activity of T-cells, thus enhancing the cellular immunity. Related studies have demonstrated that $\mathrm{CD} 4^{+}$ T-cells play an important role in resisting the pathogens that evade MHC-I processing pathway in acute or chronic infectious diseases [33]. In addition, when $\mathrm{CD}^{+} \mathrm{T}$-cells and irradiated peripheral blood mononuclear cells were cocultivated, some antiviral bioactive substances were found in cell culture supernatant [34]. Therefore, the increasing counts of $\mathrm{CD}^{+}$and $\mathrm{CD} 8^{+}$T-cells will enhance the body's immune and antiviral level. Our previous studies also proved that some effective constituents from plant could improve the proportions of T-lymphocytes subset when encapsulated with liposome $[26,35]$.

Cytokine is one kind of nonspecific immunoreactive product [36]. IFN- $\gamma$ could promote the proliferation and differentiation of T-cells, activate macrophages, and enhance the cytotoxic effect of NK cells. IL- 6 could induce the proliferation and differentiation of B-cells, promote the generation of antibody, and thus mediate humoral immunity $[37,38]$. Based on the immunomodulatory effects of IFN- $\gamma$ and IL6 , the effect of EPL on the secretion of IFN- $\gamma$ and IL- 6 was 


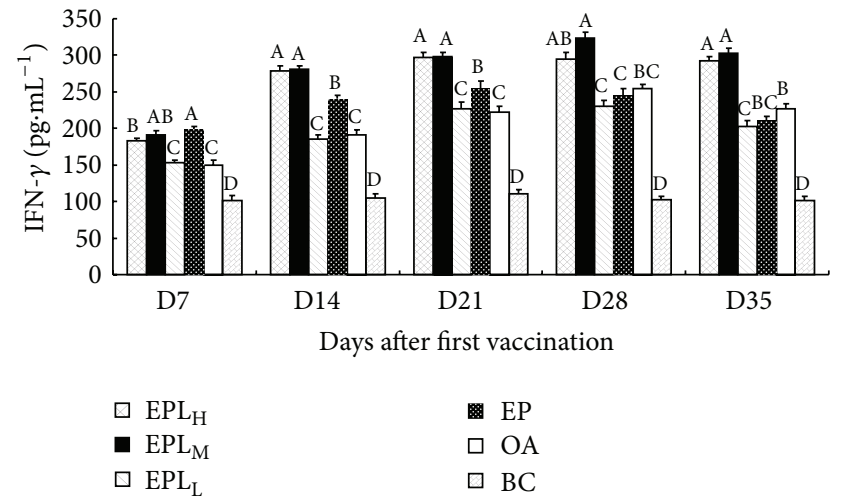

(a)
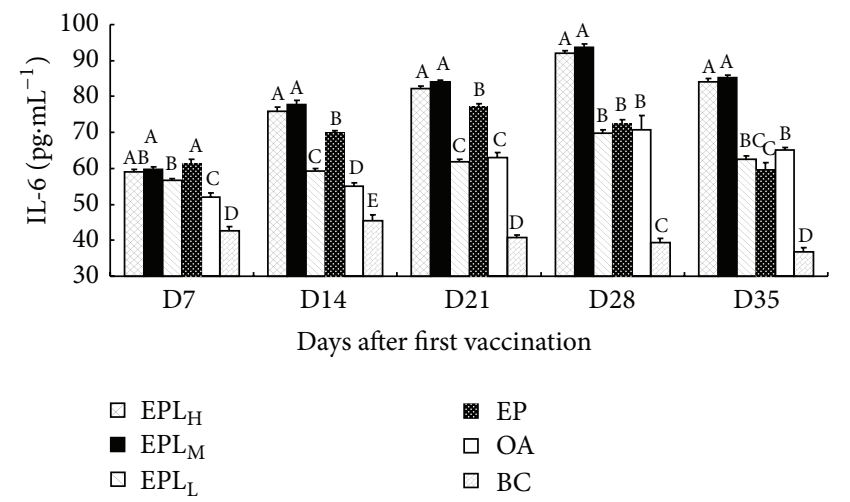

(b)

FIGURE 4: The effects of EPI on the secretion of cytokines in the immunized mice (pg. $\mathrm{mL}^{-1}$ ). (a) The changes of serum IFN- $\gamma$ contents. (b) The changes of serum IL- 6 contents. Bars in the same day without the same superscripts differ significantly $(P<0.05)$. H: high dose; M: medium dose; L: low dose.

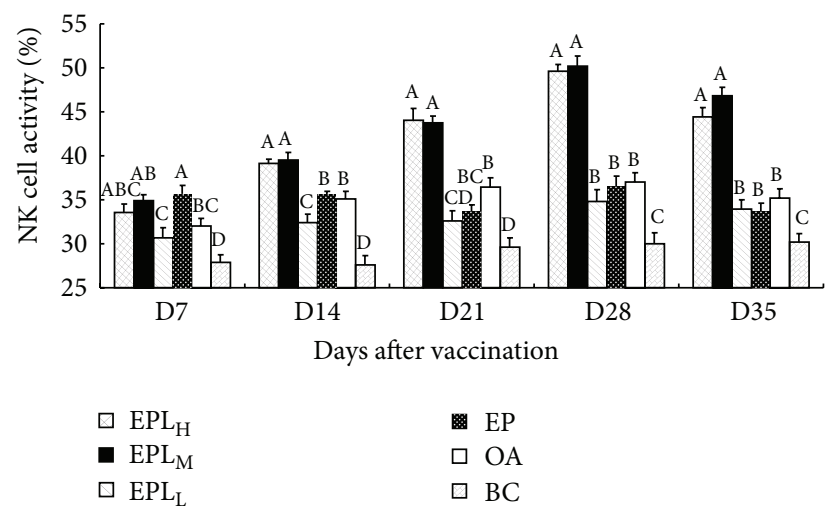

FIGURE 5: Effect of EPL on the activity of NK cells. Bars in the same day without the same superscripts differ significantly $(P<0.05) . \mathrm{H}$ : high dose; M: medium dose; L: low dose.

measured in this study. The array data showed that EPL could significantly promote the mRNA expression levels of IFN- $\gamma$ and IL- 6 in vitro. In addition, the contents of IFN$\gamma$ and IL-6 in EPL high and medium dosage groups were significantly higher than those in EP group on days 14-35. This suggested that EPL was capable of inducing a large pool of cytokines, and the effect was significantly better than EP. The supplement of EPL in the vaccine is a benefit to improve the immunologic response of body against PCV2.

NK cells are one kind of independent lymphocyte population; they could kill target cells without prior sensitization by antigen. NK cells are the important components of innate immune system and are the first line of defense against the infection of pathogenic microorganism and tumor [39]. Besides killing target cells, NK cells could also release a variety of cytokines (e.g., TNF and IFN- $\gamma$ ), which play important roles in the immune regulation and the differentiation of immune cells [40]. In this investigation, the results showed that EPL at high and medium doses and EP could significantly increase the killing activity of NK cells in the immunized mice, and the effect of EPL was significantly superior to EP, suggesting that the usage of EPL in PCV2 vaccine can help to improve the nonspecific immunity to kill virus. Sun et al. also proved that Albizia julibrissin saponins from plant could enhance the killing activity of NK cells in the OVAimmunized mice, which is similar to our results [41].

IgG is one of the important immunoglobulins produced in secondary humoral immune response, which possesses various activities, such as antibacterial, antivirus, and antiexotoxin activities. Therefore, the content of IgG not only represents the antibody activity, but also is the characterization reflected by the humoral immune function of body [42]. There are different IgG subclasses including IgG1, $\operatorname{IgG} 2 \mathrm{a}, \operatorname{IgG} 2 \mathrm{~b}$, and IgG3. The production of IgG subclasses is regulated by T-lymphocytes and their cytokines. IL- 4 and IL-6 produced by Th2 lymphocytes improve the production of IgG1, while Thl cytokines such as IL-2 and IFN$\gamma$ improve the secretion of IgG2a [43]. According to the immune response of body against the infectious diseases, the Th1 response mainly targets intracellular pathogens, while the Th2 response primarily targets extracellular pathogens $[43,44]$. Data in Figure 6 indicated that the EPL could significantly improve the titers of IgG, IgG1, and IgG2a at most time points compared with EP. Enhanced production of IgG and subclasses may be explained by increased secretion of both IFN- $\gamma$ and IL- 6 as shown in Figure 4 . All these suggested that EPL as an adjuvant could upregulate both Th1 and Th2 immune responses.

PMWS is the serious viral disease and causes a major threat to the pig-breeding industry. Therefore, the development of new efficient adjuvants for PCV2 vaccine is of special urgency. In this study, our results confirmed that EPL could improve the immune response of the inactivated PCV2 vaccine, and the adjuvant effect was significantly better than OA, especially in cellular immunity. In addition, EPL had no side effect, which was safe. Therefore, EPL should be an ideal adjuvant candidate for developing a new type of PCV2 vaccine. 


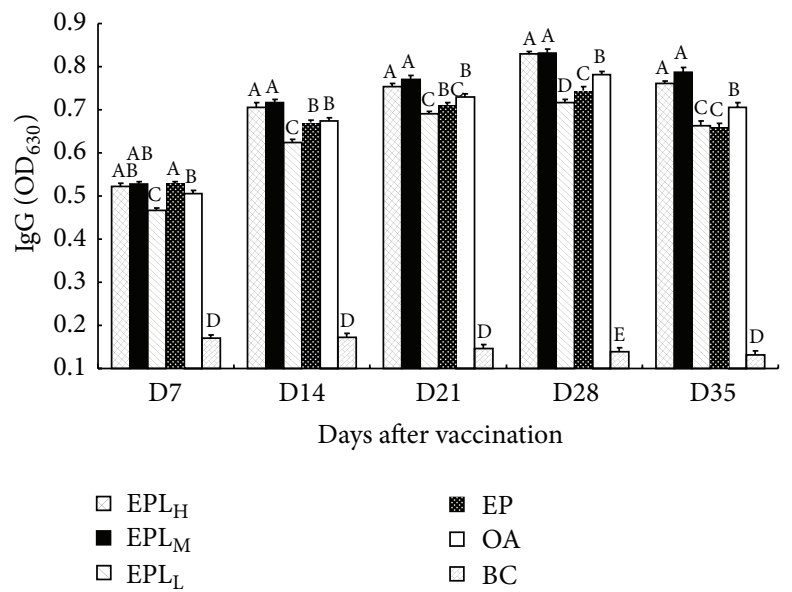

(a)

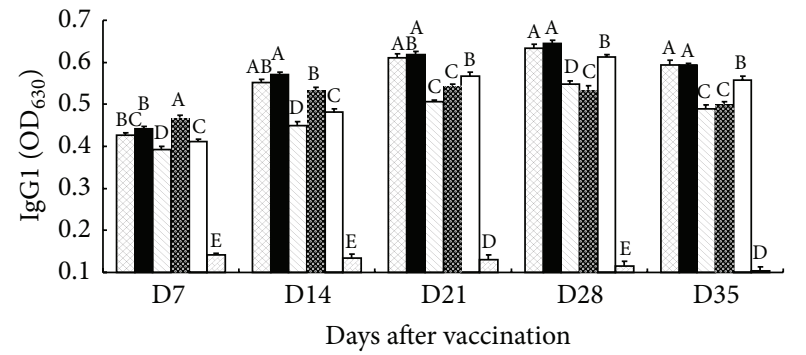

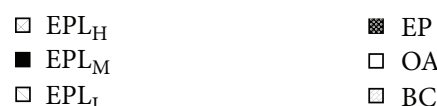

(b)
网 $\mathrm{EP}$
$\square \mathrm{OA}$
$\square \mathrm{BC}$

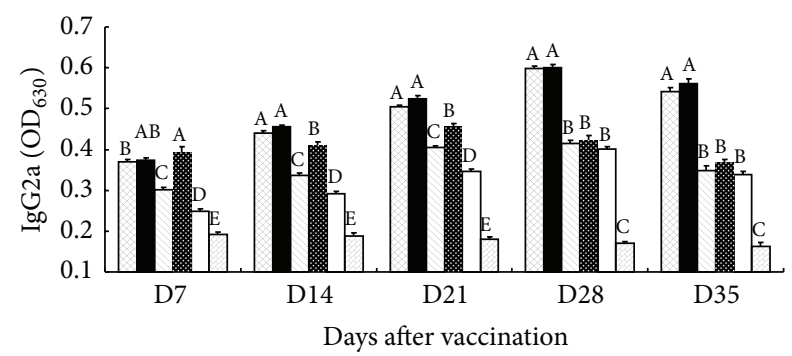
$\square \mathrm{EPL}_{\mathrm{H}}$
圆 EP
- $\mathrm{EPL}_{\mathrm{M}}$
$\square \mathrm{EPL}_{\mathrm{L}}$
$\square \mathrm{OA}$
․ BC

(c)

Figure 6: Effect of EPL on PCV2-specific IgG, IgG1, and IgG2a antibodies in the immunized mice. (a) The titer of IgG. (b) The titer of IgG2a. (c) The titer of IgG2a. Bars in the same day without the same superscripts differ significantly $(P<0.05)$. H: high dose; M: medium dose; L: low dose.

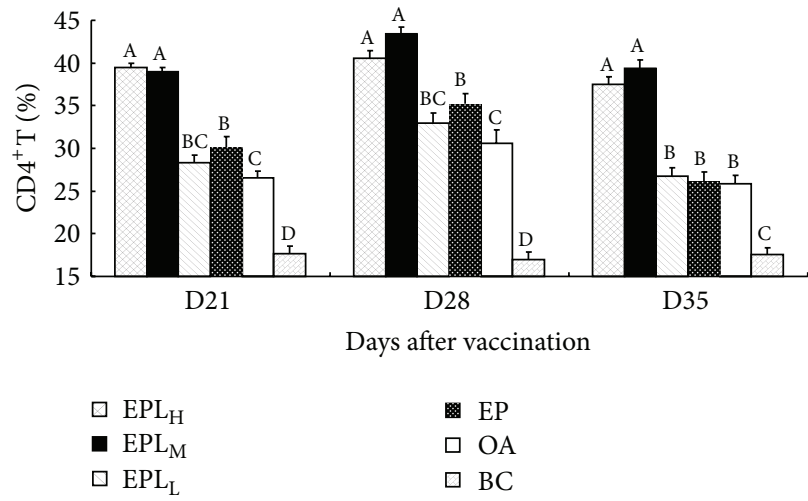

(a)

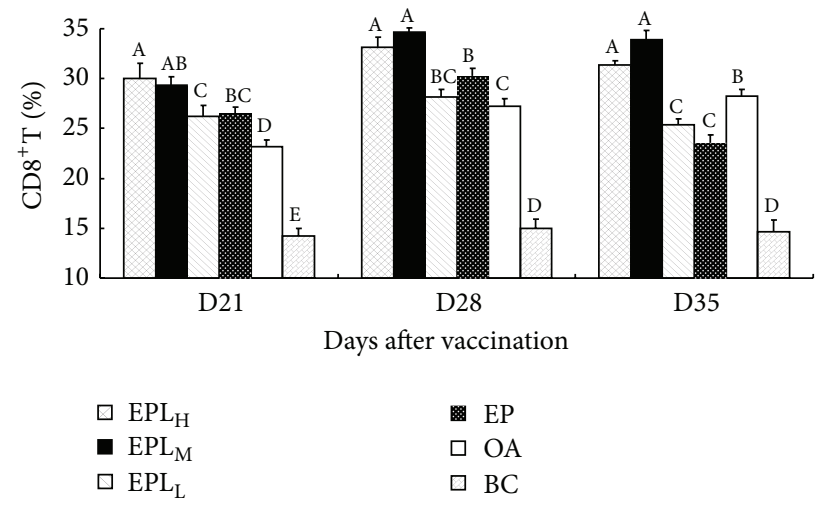

(b)

FIgure 7: The effects of EPI on the percentage of $\mathrm{CD} 4^{+}$and $\mathrm{CD}^{+}$T-cells in the immunized mice. (a) The changes of CD4 ${ }^{+} \mathrm{T}$-cells. (b) The changes of $\mathrm{CD}^{+}$T-cells. Bars in the same day without the same superscripts differ significantly $(P<0.05)$. H: high dose; M: medium dose; L: low dose. 


\section{Conclusions}

The present study demonstrated that EPL possessed better adjuvant activity in inducing the specific cellular and humoral immune responses in mice. These findings indicated that EPL may be a promising adjuvant to the inactivated PCV2 vaccine by providing higher and long lasting protective effect. To understand the potential of EPL in clinical applications as adjuvant, further studies on the mechanism of action are in progress.

\section{List of Abbreviations}

PCV: Porcine circovirus

PMWS: Postweaning multisystemic wasting syndrome

EPL: Epimedium polysaccharide-propolis flavone liposome

EP: $\quad$ Epimedium polysaccharide-propolis flavone

MTT: 3-(4,5-Dimethylthiazol-2-yl)-2,5-diphenyltetrazolium bromide

PBS: $\quad$ Phosphate-buffered saline

PHA: Phytohemagglutinin

LPS: Lipopolysaccharide

DMSO: Dimethyl sulfoxide

BL: Blank liposome

CC: $\quad$ Cell control

OA: Oil adjuvant

BC: Blank control.

\section{Conflict of Interests}

None of the authors has any financial or personal competing interests that could inappropriately influence or bias the content of the paper.

\section{Authors' Contribution}

Yunpeng Fan and Liwei Guo contributed equally to this work. Yunpeng Fan designed the study and experiments, interpreted the experimental data, and drafted the paper. Liwei Guo, Weifeng Hou, and Chao Guo performed the experiments. Weimin Zhang, Xia Ma, and Lin Ma contributed to samples collection. Xiaoping Song helped in data analysis. Yunpeng Fan delivered the paper draft. All authors read and approved the final paper.

\section{Acknowledgments}

This research was supported by the National Natural Science Foundation of China (Grant no. 31402240), Fundamental Research Funds for the Central Universities (Grant no. 2452015037), and Project Funded by China Postdoctoral Science Foundation (Grants nos. 2015T81061, 2014M550516).

\section{References}

[1] Z. Yin and J. H. Liu, Animal Virology, Science Press, Beijing, China, 1997.
[2] J. Segales, C. Piñeiro, F. Lampreave et al., "Haptoglobin and pigmajor acute protein are increased in pigs with postweaning multisystemic wasting syndrome (PMWS)," Veterinary Research, vol. 35, no. 3, pp. 275-282, 2004.

[3] J. A. Ellis, A. Bratanich, E. G. Clark et al., "Coinfection by porcine circoviruses and porcine parvovirus in pigs with naturally acquired postweaning multisystemic wasting syndrome," Journal of Veterinary Diagnostic Investigation, vol. 12, no. 1, pp. 21-27, 2000.

[4] J. C. S. Harding, "The clinical expression and emergence of porcine circovirus 2," Veterinary Microbiology, vol. 98, no. 2, pp. 131-135, 2004.

[5] C. Chae, "A review of porcine circovirus 2-associated syndromes and diseases," Veterinary Journal, vol. 169, no. 3, pp. 326336, 2005.

[6] L. Zhao, A. Seth, N. Wibowo et al., "Nanoparticle vaccines," Vaccine, vol. 32, no. 3, pp. 327-337, 2014.

[7] J. C. Aguilar and E. G. Rodríguez, "Vaccine adjuvants revisited," Vaccine, vol. 25, no. 19, pp. 3752-3762, 2007.

[8] S. G. Reed, S. Bertholet, R. N. Coler, and M. Friede, "New horizons in adjuvants for vaccine development," Trends in Immunology, vol. 30, no. 1, pp. 23-32, 2009.

[9] A. M. Harandi, J. Brewer, and V. Schijns, "Conference scene: Recent advancements in immunopotentiators for modern vaccines," Immunotherapy, vol. 3, no. 11, pp. 1297-1301, 2011.

[10] P. V. Licciardi and J. R. Underwood, "Plant-derived medicines: a novel class of immunological adjuvants," International Immunopharmacology, vol. 11, no. 3, pp. 390-398, 2011.

[11] K. Nada, Č. Miodrag, B. Aleksandar, and D. K. Zvezdana, "Immunomodulatory effects of the methanolic extract of Epimedium alpinum in vitro," Fitoterapia, vol. 77, no. 7-8, pp. 561-567, 2006.

[12] Z. Q. Shen and Y. F. Yang, "Propolis, a new adjuvant," Chinese Journal of Preventive Veterinary Medicine, vol. 5, pp. 55-57, 1989.

[13] Y. P. Fan, Y. L. Hu, D. Y. Wang et al., "Epimedium polysaccharide and propolis flavone can synergistically stimulate lymphocyte proliferation in vitro and enhance the immune responses to ND vaccine in chickens," International Journal of Biological Macromolecules, vol. 47, no. 2, pp. 87-92, 2010.

[14] Y. P. Fan, D. Y. Wang, J. G. Liu et al., "Adjuvanticity of epimedium polysaccharide-propolis flavone on inactivated vaccines against AI and ND virus," International Journal of Biological Macromolecules, vol. 51, no. 5, pp. 1028-1032, 2012.

[15] Y. P. Patil and S. Jadhav, "Novel methods for liposome preparation," Chemistry and Physics of Lipids, vol. 177, pp. 8-18, 2014.

[16] J. Yuan, J. G. Liu, Y. L. Hu et al., "The immunological activity of propolis flavonoids liposome on the immune response against ND vaccine," International Journal of Biological Macromolecules, vol. 51, no. 4, pp. 400-405, 2012.

[17] H. Gao, Y. P. Fan, D. Y. Wang et al., "Optimization on preparation condition of epimedium polysaccharide liposome and evaluation of its adjuvant activity," International Journal of Biological Macromolecules, vol. 50, no. 1, pp. 207-213, 2012.

[18] Y. P. Fan, D. Y. Wang, Y. L. Hu et al., "Liposome and epimedium polysaccharide-propolis flavone can synergistically enhance immune effect of vaccine," International Journal of Biological Macromolecules, vol. 50, no. 1, pp. 125-130, 2012.

[19] Y. P. Fan, J. G. Liu, D. Y. Wang et al., “The preparation optimization and immune effect of epimedium polysaccharide-propolis flavone liposome," Carbohydrate Polymers, vol. 94, no. 1, pp. 2430, 2013. 
[20] B. Q. Liu, Veterinary Biology Produce, Chinese Agricultural Press, Beijing, China, 1999.

[21] Y. Huang, C. H. Wu, Z. G. Liu et al., "Optimization on preparation conditions of Rehmannia glutinosa polysaccharide liposome and its immunological activity," Carbohydrate Polymers, vol. 104, no. 1, pp. 118-126, 2014.

[22] M. M. O. Thekisoe, P. A. Mbati, and S. P. R. Bisschop, "Different approaches to the vaccination of free ranging village chickens against Newcastle disease in Qwa-Qwa, South Africa," Veterinary Microbiology, vol. 101, no. 1, pp. 23-30, 2004.

[23] K. J. Livak and T. D. Schmittgen, "Analysis of relative gene expression data using real-time quantitative PCR and the 2[delta] [delta] CT method," Methods, vol. 25, no. 4, pp. 402-408, 2001.

[24] H.-X. Sun, H. Wang, H.-S. Xu, and Y. Ni, "Novel polysaccharide adjuvant from the roots of Actinidia eriantha with dual Thl and Th2 potentiating activity," Vaccine, vol. 27, no. 30, pp. 3984-3991, 2009.

[25] H. X. Sun, L. Q. Chen, J. Wang, K. Wang, and J. Zhou, "Structure-function relationship of the saponins from the roots of Platycodon grandiflorum for hemolytic and adjuvant activity," International Immunopharmacology, vol. 11, no. 12, pp. 20472056, 2011.

[26] Y. P. Fan, X. Ma, J. Zhang et al., “Ophiopogon polysaccharide liposome can enhance the non-specific and specific immune response in chickens," Carbohydrate Polymers, vol. 119, pp. 219227, 2015.

[27] Y. Yu, D. Wang, S. Abula et al., "The immunological adjuvant activity of gypenosides liposome against Newcastle disease vaccine," International Journal of Biological Macromolecules, vol. 60, pp. 116-121, 2013.

[28] A. S. McKee, M. K. L. MacLeod, J. W. Kappler, and P. Marrack, "Immune mechanisms of protection: can adjuvants rise to the challenge?” BMC Biology, vol. 8, article 34, 2010.

[29] X. Su, Z. Pei, and S. Hu, "Ginsenoside Re as an adjuvant to enhance the immune response to the inactivated rabies virus vaccine in mice," International Immunopharmacology, vol. 20, no. 2, pp. 283-289, 2014.

[30] J. Zhu and W. E. Paul, "CD4 T cells: fates, functions, and faults," Blood, vol. 112, no. 5, pp. 1557-1569, 2008.

[31] D. M. Brown, "Cytolytic CD4 cells: direct mediators in infectious disease and malignancy," Cellular Immunology, vol. 262, no. 2, pp. 89-95, 2010.

[32] S. F. Li, Y. Li, J. D. Zhang, C. S. Zheng, and F. Sun, "Effects of rice bran polysaccharide on $\mathrm{CD}^{+}{ }^{+} \mathrm{T}$ cells and $\mathrm{CD} 8^{+} \mathrm{T}$ cells in chickens," Animal Husbandry and Veterinary Medicine, vol. 4, pp. 59-62, 2009.

[33] D. M. Brown, C. Kamperschroer, A. M. Dilzer, D. M. Roberts, and S. L. Swain, "IL-2 and antigen dose differentially regulate perforin- and FasL-mediated cytolytic activity in antigen specific $\mathrm{CD}^{+}{ }^{+} \mathrm{T}$ cells," Cellular Immunology, vol. 257, no. 1-2, pp. 69-79, 2009.

[34] J. A. Levy, "The search for the CD8+ cell anti-HIV factor (CAF)," Trends in Immunology, vol. 24, no. 12, pp. 628-632, 2003.

[35] X. J. Zhao, Y. P. Fan, D. Y. Wang et al., "Immunological adjuvant efficacy of glycyrrhetinic acid liposome against Newcastle disease vaccine," Vaccine, vol. 29, no. 52, pp. 9611-9617, 2011.

[36] J. L. Meng, C. P. Feng, H. Y. Cheng, M. C. Chang, J. Wang, and H. J. Li, "Effects of Agaricus blazei murrill polysaccharide on the mRNA expression of the cytokines in rats," Journal of Chinese
Institute of Food Science and Technology, vol. 12, no. 4, pp. 1924, 2012.

[37] Y. Huang, C. M. Jiang, Y. L. Hu et al., "Immunoenhancement effect of rehmannia glutinosa polysaccharide on lymphocyte proliferation and dendritic cell," Carbohydrate Polymers, vol. 96, no. 2, pp. 516-521, 2013.

[38] A. S. McKee, M. W. Munks, and P. Marrack, "How do adjuvants work? important considerations for new generation adjuvants," Immunity, vol. 27, no. 5, pp. 687-690, 2007.

[39] N. Matsushita, A. Aruga, Y. Kobayashi, K. Tanigawa, and M. Yamamoto, "Separation methods of T cells, natural killer, and dendritic cells from peripheral blood of cancer patients using interleukin-2 and functional analysis of natural killer cells after separation," Immunopharmacology and Immunotoxicology, vol. 29, no. 1, pp. 31-47, 2007.

[40] M. Ohira, H. Ohdan, H. Mitsuta et al., "Adoptive transfer of TRAIL-expressing natural killer cells prevents recurrence of hepatocellular carcinoma after partial hepatectomy," Transplantation, vol. 82, no. 12, pp. 1712-1719, 2006.

[41] H. X. Sun, S. W. He, and M. H. Shi, "Adjuvant-active fraction from Albizia julibrissin saponins improves immune responses by inducing cytokine and chemokine at the site of injection," International Immunopharmacology, vol. 22, no. 2, pp. 346-355, 2014.

[42] L. Yuan, L. Wu, J. Chen, Q. Wu, and S. H. Hu, "Paclitaxel acts as an adjuvant to promote both Th1 and Th2 immune responses induced by ovalbumin in mice," Vaccine, vol. 28, no. 27, pp. 4402-4410, 2010.

[43] S. Kamstrup, R. San Martin, A. Doberti, H. Grande, and K. Dalsgaard, "Preparation and characterisation of quillaja saponin with less heterogeneity than Quil-A," Vaccine, vol. 18, no. 21, pp. 2244-2249, 2000.

[44] X. M. Song, L. M. Zang, and S. H. Hu, "Amplified immune response by ginsenoside-based nanoparticles (ginsomes)," Vaccine, vol. 27, no. 17, pp. 2306-2311, 2009. 


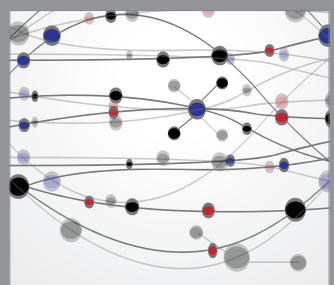

The Scientific World Journal
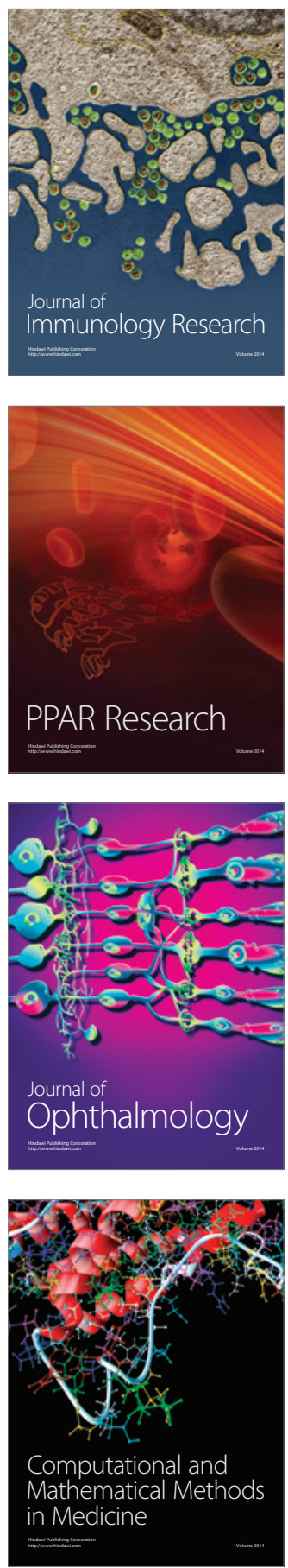

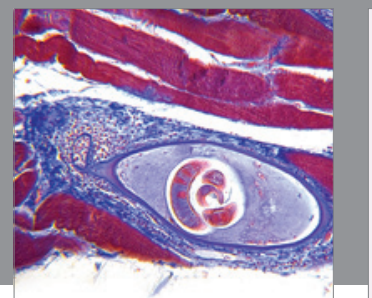

Gastroenterology

Research and Practice
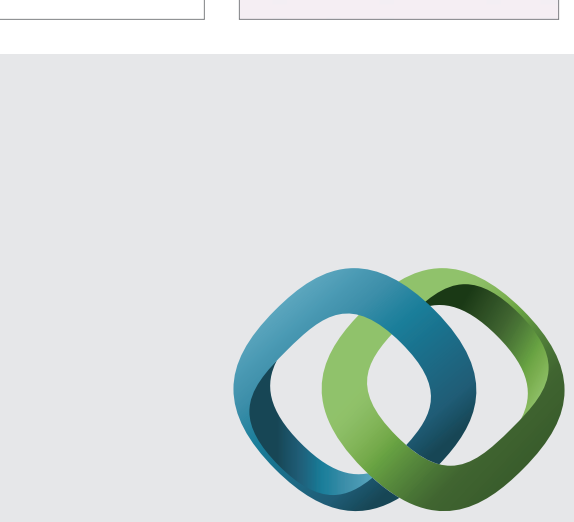

\section{Hindawi}

Submit your manuscripts at

http://www.hindawi.com
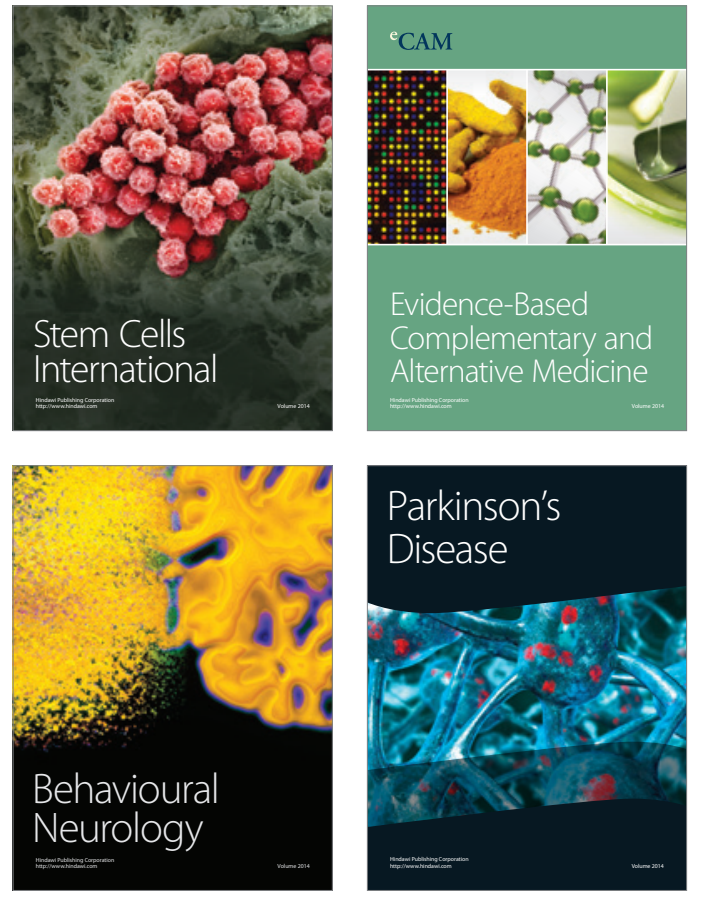
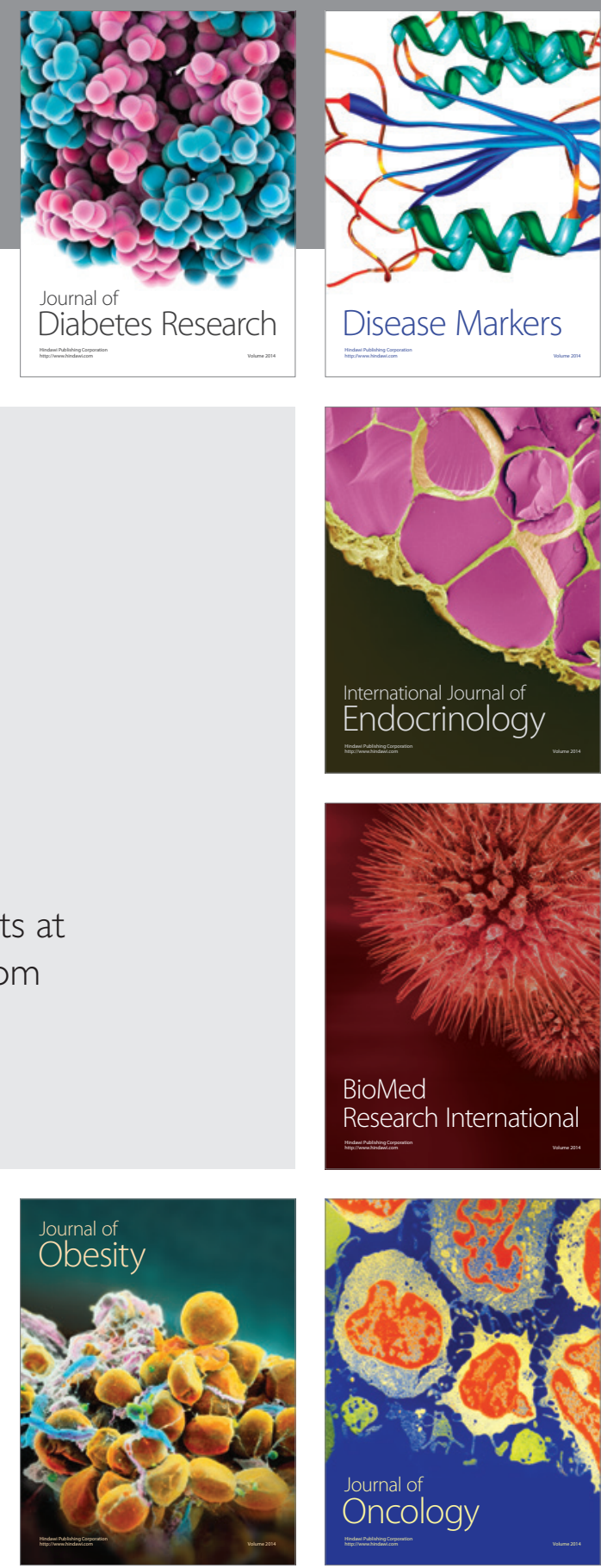

Disease Markers
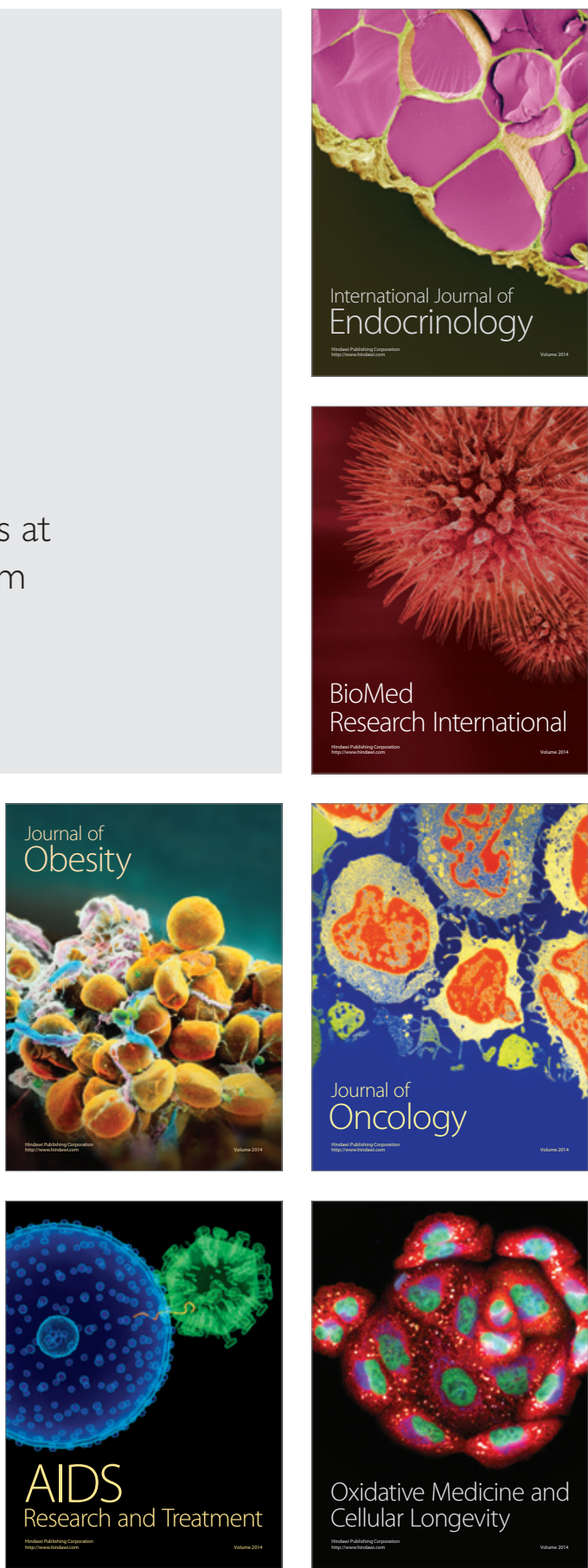